\title{
medi-notes
}

THOMAS WESLEY ALLEN, D.O.

Editor in Chief

GEORGE W. NORTHUP, D.o.

Editor Emeritus

\section{Colitis in the older patient}

In the elderly, colitis is a heterogeneous group of disorders. Each type has its own historic features and treatment, but each may mimic one or more of the others or may be superimposed on another.

One of the most common types among older patients is infectious colitis, which may be caused by viruses, bacteria, or parasites. The clinical, sigmoidoscopic, endoscopic, and radiologic features may mimic those of inflammatory bowel disease or even ischemic colitis. It is best diagnosed with both stool and blood cultures.

Antibiotic-associated colitis may follow treatment with almost any antibacterial agent. It usually is caused by Clostridium difficile. A careful history will help in diagnosis. Most patients recover spontaneously.

Ischemic colitis is the most common noninfectious colitis that appears after 50 years of age. Characteristic features include segmental distribution of disease, association with a normal rectum, spontaneous resolution in half of the episodes, a low incidence of recurrence, and frequent progression to fibrosis and stricture, followed later by bowel obstruction. Colonic ischemia may be associated with other large bowel lesions. Management is chiefly supportive in acute cases or surgical in chronic cases.

Inflammatory bowel disease of new onset in the elderly is more likely to be ulcerative colitis than Crohn's disease. Knowledge regarding natural history, location, response to treatment, and recurrence of these two diseases needs additional study. Diagnosis of Crohn's disease requires exclusion of infectious and ischemic colitis, neoplasm, and diverticular disease. Surgery eventually is required in about half of older patients with ulcerative colitis but in most of those with Crohn's disease. Mortality is high, especially if operation is performed on an emergency basis.

Radiation colitis may appear months or years after completion of radiotherapy. Early clinical symptoms may resemble those of infectious or ulcerative colitis, and changes visible on sigmoidoscopy or barium enema findings may resemble those of ulcerative colitis, inflammatory colitis, or localized cancer of the colon.

Patient evaluation requires a careful history and physical examination. Sigmoidoscopy is always necessary, with the choice between rigid or flexible instrumentation of little importance if the physician is aware of the advantages and limitations of each technique. Endoscopic differential diagnosis requires scrutiny for rectal involvement, submucosal hemorrhage, segmental disease, rapid resolution, and histologic changes. If diagnosis cannot be made from sigmoidoscopy, colonoscopy or barium enema may be necessary, the choice depending on the condition of the patient and the suspected disease. Biopsy section should include both diseased and normal-appearing mucosa

Brandt, L.J.: Colitis in the elderly. Hosp Practice $22: 165-88,15$ Jun 87

\section{International travel and viral hepatitis}

Viral hepatitis is a recognized risk for international travelers. The risk for hepatitis A increases with deviation from customary tourist routes, a high frequency of the disease in the region visited, and a long stay in an endemic area. Travelers who will encounter these risks are candidates for immune globulin protection be- fore their trip.

Hepatitis B risk factors include prolonged residence, frequent travel, or extensive contact with local populations in endemic areas. These conditions may suggest the need for hepatitis B vaccination prior to travel.

The risk of non-A, non-B hepatitis may be greater overseas. Travelers should be warned to avoid transfusions outside the United States unless they are necessary for life. The average international traveler is at least risk for delta hepatitis, but would be protected against it by hepatitis $B$ vaccine. However, physicians should warn carriers of hepatitis B surface antigen not to travel to regions with an epidemic of delta hepatitis or with high delta endemicity.

Lange, W.R.: Viral hepatitis and international travel. Am Fam Physician 36:179-84, Jul 87

\section{Selection of lipid-lowering agents}

Lipid abnormalities usually respond well to treatment with diet alone. However, many patients with hyperlipidemia also need lipid-lowering drugs. Such agents should be employed only if a 6-months' trial of diet therapy has failed.

This paper categorizes patients on the basis of knowledge of three primary lipid measurements: total cholesterol; total triglyceride; and highdensity lipoprotein (HDL) cholesterol. These can be used to determine which drugs to employ and what dosage to give.

Patients comprising Category A have high total cholesterol levels (more than $240 \mathrm{mg}$./dl. on two occasions), normal triglyceride values (less than $250 \mathrm{mg} . / \mathrm{dl}$.), and normal or slightly depressed HDL cholesterol levels (40 mg./dl. or less). Lipid- 
The portrait of anxiety

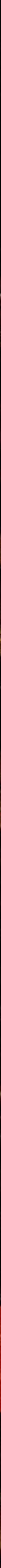




\section{is often complicated}

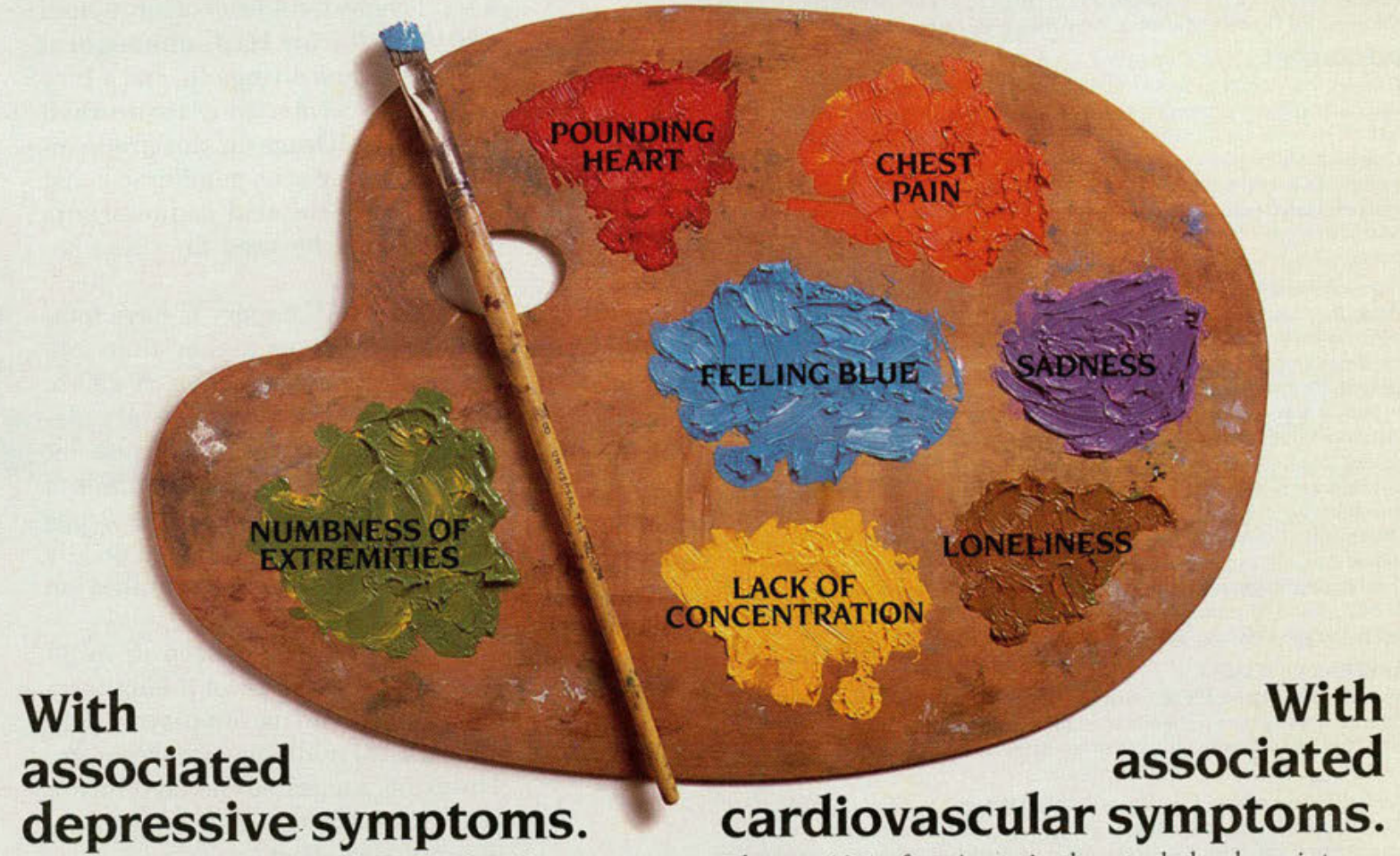

In double-blind four-week clinical trials in 632

patients with moderate to severe anxiety, therapy with XANAX was compared with placebo.

XANAX was significantly more effective $(P<.001)$ than placebo in relieving the anxiety. with over half of the patients showing marked to moderate improvement by the first evaluation period (one week).

In addition, over $70 \%$ of these patients experienced associated moderate to severe depressed mood. XANAX was shown to be significantly more effective $(P<014)$ than placebo in improving the associated depressed mood.

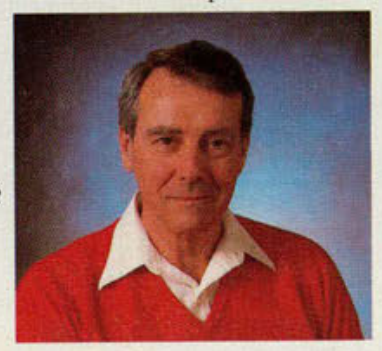

Almost $60 \%$ of patients in the study had anxiety with associated cardiovascular symptoms even though cardiovascular disease had been ruled out. XANAX was shown to effectively relieve anxiety including the associated cardiovascular symptoms. XANAX, the first of a unique class-the triazolobenzodiazepines.

- Well tolerated-Side effects, if they occur are generally observed at the beginning of therapy and usually disappear with continued medication. Drowsiness and light-headedness were the most commonly reported adverse reactions.

- Sustained efficacy-No reported increase in dosage during 16-week clinical study, once an appropriate dosage was achieved. Since long-term effectiveness of XANAX has not been established, it is recommended that it not be used for longer than 16 weeks.

Simple dosage -0.25 to $0.5 \mathrm{mg}$ ti.d.
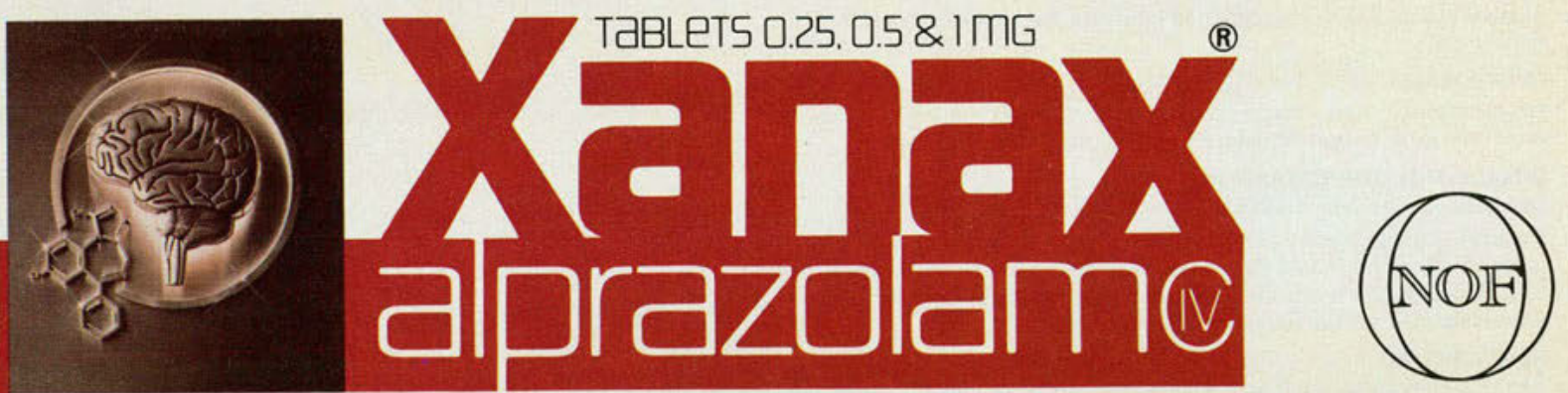

\section{for the relief of complicated anxiety}




\section{XANAX Tablets \\ (alprazolam) 6}

\section{CONTRAINDICATIONS}

Patients with sensitivity to this drug or other benzodiazepines and in acute narrow angle glaucoma

\section{WARNINGS}

Not of value in psychotic patients. Caution patients against hazardous occupations requiring complete mental alertness and about the simultaneous ingestion of alcohol and other CNS depressant drugs.

Benzodiazepines can cause fetal harm in pregnant women. Warn patients of the potential hazard to the fetus. Avoid during the first trimester.

\section{PRECAUTIONS}

General: The dosage of XANAX Tablets should be reduced or withdrawn gradually, since withdrawal seizures have been reported upon abrupt withdrawal. If XANAX is combined with other psychotropics or anticonvulsant drugs, consider drug potentiation (see Drug Interaction section). Exercise the usual precautions regarding size of the prescription for depressed or suicidal patients. In elderly and debilitated patients, use the lowest possible dosage (see Dosage and Administration). Observe the usual precautions in treating patients with impaired renal or hepatic function.

Information for Patients: Alert patients about: (a) consumption of alcohol and drugs, (b) possible fetal abnormalities, (c) operating machinery or driving, (d) not increasing dose of the drug due to risk of dependence, (e) not stopping the drug abruptly. Laboratory Tests: Not ordinarily required in otherwise healthy patients. Drug Interactions: Additive CNS depressant effects with other psychotropics, anticonvulsants, antihistamines, ethanol and other CNS depressants. Pharmacokinetic interactions with other drugs, e.g., cimetidine, have been reported. Drug/Laboratory Test Interactions: No consistent pattern for a drug or test Carcinogenesis, Mutagenesis, Impairment of Fertility: No carcinogenic potential or impairment of fertility in rats. Pregnancy: See Warnings. Nonteratogenic Effects: The child born of a mother on benzodiazepines may be at some risk for withdrawal symptoms and neonatal flaccidity. Labor and Delivery: No established use. Nursing Mothers: Benzodiazepines are excreted in human milk. Women on XANAX should not nurse. Pediatric Use: Safety and effectiveness in children below the age of 18 have not been established.

\section{ADVERSE REACTIONS}

Side effects are generally observed at the beginning of therapy and usually disappear with continued medication. In the usual patient, the most frequent side effects are likely to be an extension of the pharmacologic activity of XANAX, e.g., drowsiness or lightheadedness.

Central nervous system: Drowsiness, lightheadedness, depression, headache, confusion, insomnia, nervousness, syncope, dizziness, akathisia, and tiredness/ sleepiness. Gastrointestinal: Dry mouth, constipation, diarrhea, nausea/vomiting, and increased salivation. Cardiovascular: Tachycardia/palpitations, and hypotension. Sensory: Blurred vision. Musculoskeletal: Rigidity and tremor. Cutaneous: Dermatitis/allergy. Other side effects: Nasal congestion, weight gain, and weight loss. Withdrawal seizures have been reported upon rapid decrease or abrupt discontinuation of XANAX. (See Precautions.)

In addition, the following adverse events have been reported with the use of benzodiazepines: dystonia, irritability, concentration difficulties, anorexia, transient amnesia or memory impairment, loss of coordination, fatigue, sedation, slurred speech, jaundice, musculoskeletal weakness, pruritus, diplopia, dysarthria, changes in libido, menstrual irregularities, incontinence and urinary retention. Paradoxical reactions such as stimulation, agitation, increased muscle spasticity sleep disturbances, and hallucinations may occur. Should these occur, discontinue the drug.

During prolonged treatment, periodic blood counts, urinalysis, and blood chemistry analysis are advisable. Minor EEG changes, of unknown significance, have been observed.

\section{DRUG ABUSE AND DEPENDENCE}

Physical and Psychological Dependence: Withdrawal symptoms have occurred following abrupt discontinuance of benzodiazepines. Withdrawal seizures have occurred upon rapid decrease or abrupt discontinuation of therapy. In all patients, dosage should be gradually tapered under close supervision. Patients with a history of seizures or epilepsy should not be abruptly withdrawn from XANAX. Addiction-prone individuals should be under careful surveillance. Controlled Substance Class: XANAX is a controlled substance and has been assigned to schedule IV.

\section{OVERDOSAGE}

Manifestations include somnolence, confusion, impaired coordination, diminished reflexes and coma. No delayed reactions have been reported.

\section{DOSAGE AND ADMINISTRATION}

Dosage should be individualized.

The usual starting dose is 0.25 to $0.5 \mathrm{mg}$, t.i.d. Maximum total daily dose is $4 \mathrm{mg}$ In the elderly or debilitated, the usual starting dose is $0.25 \mathrm{mg}$, two or three times daily. Reduce dosage gradually when terminating therapy, by no more than 0.5 milligram every three days.

\section{HOW SUPPLIED}

XANAX Tablets are available as $0.25 \mathrm{mg}, 0.5 \mathrm{mg}$, and I $\mathrm{mg}$ tablets.

Caution: Federal law prohibits dispensing without prescription. lowering agents for this group include a combination of cholestyramine or colestipol plus any one of these four: nicotinic acid; probucol; neomycin; or lovastatin. Another recommended combination is nicotinic acid plus neomycin.

Category B includes patients with a total cholesterol level of more than $240 \mathrm{mg} . / \mathrm{dl}$., an HDL cholesterol value less than $40 \mathrm{mg}$./dl., and a very high triglyceride value (more than $500 \mathrm{mg}$./dl.). Drugs for this group include nicotinic acid, gemfibrozil, and clofibrate. Bile acid sequestrants should never be used for these patients.

Patients in Category $\mathrm{C}$ have total cholesterol values higher than 240 $\mathrm{mg} . / \mathrm{dl}$., triglyceride levels of 250 to $500 \mathrm{mg} . / \mathrm{dl}$., and moderately depressed HDL cholesterol values (at or less than $40 \mathrm{mg}$./dl.). If diet fails to alleviate these problems, the drugs used for category B patients or bile acid sequestrants in combination may be employed.

Physicians should keep in mind that drug therapy is on a long-term basis. It should be employed only after a trial of dietary intervention. The drugs suggested should be given at the lowest dosage that will achieve the intended effect.

The authors recommend that patients who fail to respond to druglowering treatment should be referred to a specialized lipid center. There, more sophisticated evaluation and therapy may be administered.

LaRosa, J.C.: Drug therapy for lipid disorders. Selecting appropriate lipid-lowering agents. Postgrad Med 82:103-11, Jul 87 